\title{
Reducing heterotic $M$-theory to five dimensional supergravity on a manifold with boundary
}

\author{
Ian G. Mos: ${ }^{*}$ \\ School of Mathematics and Statistics, Newcastle University, NE1 7RU, UK \\ John T. Omotan讨 and Paul M. Saffin用 \\ School of Physics and Astronomy, University of Nottingham, NG7 2RD, UK
}

(Dated: June 20, 2013)

\begin{abstract}
This paper constructs the reduction of heterotic $M$-theory in eleven dimensions to a supergravity model on a manifold with boundary in five dimensions using a Calabi-Yau three-fold. New results are presented for the boundary terms in the action and for the boundary conditions on the bulk fields. Some general features of dualisation on a manifold with boundary are used to explain the origin of some topological terms in the action. The effect of gaugino condensation on the fermion boundary conditions leads to a 'twist' in the chirality of the gravitino which can provide an uplifting mechanism in the vacuum energy to cancel the cosmological constant after moduli stabilisation.
\end{abstract}

\section{INTRODUCTION}

Heterotic $M$-theory [1, 2] is based on the idea that one of the low-energy limits of $M$-theory can be formulated as 11-dimensional supergravity with matter fields living on two halves of a 10-dimensional boundary. The particle content and interactions of the theory, when reduced to four dimensions, were first studied in the mid-1990's 3, 4], and have been further developed in the past few years [5 [8]. There has been considerable progress also in fixing the moduli of the theory whilst retaining small values of the cosmological constant [9-12].

An interesting feature of heterotic $M$-theory is the existence of a natural five-dimensional reduction which retains the boundary-bulk structure 13, 14]. This version of the theory plays an important role in some of the moduli stabilisation mechanisms 9 , 11 .

It is not widely appreciated that the original formulation of Hořava and Witten contained some serious problems, which limited the range of validity of their 11-dimensional theory. The source of these problems lay in the presence of distributions in the supersymmetry transformations and in the Bianchi identity of the antisymmetric tensor flux. These terms prevented construction of a supersymmetric action beyond the leading terms in an expansion parameter $\kappa_{11}^{2 / 3}$, where $\kappa_{11}$ is the gravitational coupling. These problems have now been resolved by a simple modification to the boundary conditions of the theory, allowing the supersymmetry transformations and the Bianchi identity to remain free of distributions and resulting in a low energy theory which is supersymmetric to all orders in $\kappa_{11}{ }^{2 / 3}$ [15 17].

In many cases it is possible to make progress starting from the original theory of Harava and Witten, since the modified boundary conditions have little effect on the Yukawa couplings, or on the bosonic sector per se, where most of the phenomenology resides. However, the new boundary conditions contain fermion bilinears, and one place where these become important is in the presence of a gaugino condensate. There are ways to sidestep some of the problems in the original formulation of heterotic $M$-theory to cover gaugino condensation [18], but these tricks become unnecessary when working with the correct version of the theory, and in some cases the original theory gives incorrect potentials in four dimensions 19].

The purpose of the present paper is to re-examine the five-dimensional limit of heterotic $M$-theory reduced on a Calabi-Yau three-fold. The original work on this reduction by Lukas et al [14] started from the bosonic sector of an $E_{6} \times E_{8}$ theory and constructed the bulk-fermion sector from the known five-dimensional supergravity models. This approach cannot be used on the boundary, where the bulk fields appear in the boundary action, and of course it says nothing about the boundary conditions. We shall construct the Lagrangians and boundary conditions for the $E_{6} \times E_{8}$ theory, mostly by direct reduction from 11 dimensions, resorting to supersymmetry only when the technical complexity becomes too daunting.

There are two reasons why we are interested in the five-dimensional limit of heterotic $M$-theory:

\footnotetext{
*Electronic address: ian.moss@ncl.ac.uk

${ }^{\dagger}$ Electronic address: ppxjto@nottingham.ac.uk

${ }^{\ddagger}$ Electronic address: paul.saffin@nottingham.ac.uk
} 
- Starting from supergravity on a manifold with boundary in eleven dimensions gives us a new theory of supergravity on a manifold with boundary in five dimensions.

- When there is a gaugino condensate, the fermion boundary conditions break the supersymmetry in five dimensions and lead to a mechanism for cancelling the cosmological constant.

Supergravity theories with boundary matter and dimension less than eleven have been constructed only recently. The most detailed models are those in three dimensions [20]. Examples in five [21] and seven dimensions [22] have also been constructed, but the five-dimensional model included distributions, which is something we are trying to avoid. The $E_{6} \times E_{8}$ reduction of heterotic $M$ theory leads to a new supergravity model in five dimensions with Yang-Mills and chiral matter multiplets on the boundary. We omit most of the terms with four fermion fields due to technical complexity, but we will include the four-fermion terms that are relevant to gaugino condensation. The supersymmetry of the theory is inherited from eleven dimensions, apart from one term in the supersymmetry variation which cancels the supergravity anomaly in eleven dimensions. There is no supersymmetry anomaly in five dimensions, but we can isolate the offending supersymmetry variation and regard it as a 'higher order' correction.

The gaugino condensate leads to a small chiral 'twist' between the fermions on the two separate boundary components. This creates a five dimensional vacuum energy due to quantum effects [23]. Since moduli stabilisation leads to a negative vacuum energy, the quantum vacuum energy can be used as an 'uplifting' mechanism to obtain a small positive cosmological constant in four dimensions. Previous work has considered this effect in the case of the gravitino in five dimensions [9]. We shall show that the other fermion fields are not twisted by the condensate, and consequently that the gravitino is the main contributor to the vacuum energy.

The conventions used follow Weinberg [24]. The metric signature is $-+\cdots+$. The gamma matrices satisfy $\left\{\Gamma_{I}, \Gamma_{J}\right\}=2 g_{I J}$ and $\Gamma^{I \ldots K}=\Gamma^{[I} \ldots \Gamma^{K]}$. Dirac conjugates are $\bar{\psi}=i \psi^{\dagger} \Gamma^{0}$. Index conventions are given in table 【.

TABLE I: Index conventions. The shortage of letters has meant that some indices used in section 2 have had to be re-assigned in section 3 .

\begin{tabular}{lll}
\hline \hline Index & Description & Section \\
\hline$I, J, K \ldots$ & 11-dimensional coordinates & 2 \\
$A, B, C \ldots$ & 10-dimensional coordinates & 2 \\
$N$ & outward normal & 2 \\
$\alpha, \beta, \gamma \ldots$ & 5 -dimensional coordinates & 3 \\
$\mu, \nu, \rho \ldots$ & 4-dimensional coordinates & 3 \\
$a, b, c \ldots$ & holomorphic Calabi-Yau coordinates & 3 \\
$A, B, C \ldots$ & $\mathrm{SU}(2)$ spinor indices \\
$p, q, r \ldots$ & fundamental $E_{6}$-group representation & 3 \\
$i, j, k \ldots$ & $H^{1,1}$ Calabi-Yau moduli \\
$I . J, K \ldots$ & $E_{6}$ or $E_{8}$ Lie algebra \\
$L, R$ & chiral components \\
\hline \hline
\end{tabular}

\section{THE THEORY IN ELEVEN DIMENSIONS}

We begin with some of the ingredients of the improved version of low-energy heterotic $M$-theory described in Ref. [16]. The theory is formulated on a manifold $\mathcal{M}$ with a boundary consisting of two disconnected components $\partial \mathcal{M}_{1}$ and $\partial \mathcal{M}_{2}$ with identical topology. This is sometimes called a 'downstairs' formulation, as opposed to an 'upstairs' formulation which is defined on a covering space. The supergravity multiplet is placed on $\mathcal{M}$ and Yang-Mills multiplets live on the boundary. Branes may be present, but we leave these out in the simplest version of the theory.

The eleven-dimensional part of the action is conventional for supergravity,

$$
\begin{aligned}
S_{S G}= & \frac{1}{2 \kappa_{11}^{2}} \int_{\mathcal{M}}\left(-R(\Omega)-\bar{\Psi}_{I} \Gamma^{I J K} D_{J}\left(\Omega^{*}\right) \Psi_{K}-\frac{1}{48} G_{I J K L} G^{I J K L}\right. \\
& \left.-\frac{1}{96}\left(\bar{\Psi}_{I} \Gamma^{I J K L M P} \Psi_{P}+12 \bar{\Psi}^{J} \Gamma^{K L} \Psi^{M}\right) G_{J K L M}^{*}-\frac{1}{6.11 !} \epsilon^{I_{1} \ldots I_{11}}(C \wedge G \wedge G)_{I_{1} \ldots I_{11}}\right) d v,
\end{aligned}
$$

where $G$ is the Abelian field strength and $\Omega$ is the tetrad connection. The combination $G^{*}=(G+\hat{G}) / 2$, where hats denote the standardised subtraction of gravitino terms to make a supercovariant expression. 
The boundary terms which make the action supersymmetric are

$$
S_{0}=\frac{1}{\kappa_{11}^{2}} \int_{\partial \mathcal{M}}\left(K \mp \frac{1}{4} \bar{\Psi}_{A} \Gamma^{A B} \Psi_{B}+\frac{1}{2} \bar{\Psi}_{A} \Gamma^{A} \Psi_{N}\right) d v
$$

where $K$ is the extrinsic curvature of the boundary. The fermionic additions to the extrinsic curvature term were discovered first for three dimensions in Ref. [25]. We shall take the upper sign on the boundary component $\partial \mathcal{M}_{1}$ and the lower sign on the boundary component $\partial \mathcal{M}_{2}$.

There are also boundary terms with the Yang-Mills multiplets. These are scaled, by a parameter $\epsilon$, compared to the supergravity terms,

$$
S_{1}=-\frac{\epsilon}{\kappa_{11}^{2}} \int_{\partial \mathcal{M}} d v\left(\frac{1}{4}\left(\operatorname{tr} F^{2}-\frac{1}{2} \operatorname{tr} R^{2}\right)+\frac{1}{2} \operatorname{tr} \bar{\chi} \Gamma^{A} D_{A}\left(\hat{\Omega}^{* *}\right) \chi+\frac{1}{4} \bar{\Psi}_{A} \Gamma^{B C} \Gamma^{A} \operatorname{tr} F_{B C}^{*} \chi\right),
$$

where $F^{*}=(F+\hat{F}) / 2$ and $\Omega^{* *}=\left(\Omega+\Omega^{*}\right) / 2$. The original formulation of Hořava and Witten contained an extra ' $\chi \chi \chi \Psi$ ' term, but it is not present in the new version. The formulation given in Ref. [17] was only valid to order $R$, and the extension of the theory to include the $R^{2}$ term has been reported recently [26]. In the present paper we consider a small curvature limit, and include only the $O\left(R^{2}\right)$ terms needed in (3) for reducing the theory to five dimensions. In particular, higher-order terms in the gravitino given in Ref. [26] have been omitted.

The specification of the theory is completed by boundary conditions for the tangential components of the antisymmetric tensor,

$$
C_{A B C}=\mp \epsilon\left(\omega_{A B C}^{Y}-\frac{1}{2} \omega_{A B C}^{L}\right) \mp \frac{1}{4} \epsilon \operatorname{tr} \bar{\chi} \Gamma_{A B C} \chi
$$

where $\omega^{Y}$ and $\omega^{L}$ are the Yang-Mills and Lorentz Chern-Simons forms. These boundary conditions replace the modified Bianchi identity in the old formulation. A suggestion along these lines was made in the original paper of Hořava and Witten [2]. (This boundary condition is determined by anomaly cancellation and supersymmetry. It apparently places a restriction on the Abelian symmetry, although, if we follow [27, 28], we find that fixing $C_{A B C}$ on the boundary is consistent with the BRST symmetry of the gauge-fixed action and perfectly valid.) Anomaly cancellation fixes the relative coupling of the supergravity and Yang-Mills sectors,

$$
\epsilon=\frac{1}{4 \pi}\left(\frac{\kappa_{11}}{4 \pi}\right)^{2 / 3} .
$$

Further details of the anomaly cancellation, and additional fermionic Green-Schwarz terms, can be found in Ref. [17].

Boundary conditions for the gravitino can be obtained by variation of the action,

$$
\Gamma^{A B}\left(P_{ \pm}+\epsilon \Gamma P_{\mp}\right) \Psi_{A}=\epsilon J_{Y}^{A}
$$

where $P_{ \pm}$are chiral projectors based on the outward-going normals, $J_{Y}$ is the Yang-Mills supercurrent and

$$
\Gamma=\frac{1}{96} \operatorname{tr}\left(\bar{\chi} \Gamma_{A B C} \chi\right) \Gamma^{A B C} .
$$

Higher-order gravitino terms have been omitted in accordance with our small-curvatuture approximation. (Another way to obtain the gravitino boundary condition is to integrate the Rarita-Schwinger equation across the orbifold fixed points in the 'upstairs' version of the theory [16].) Similar boundary conditions are placed on the supersymmetry parameter $\eta$,

$$
\Gamma^{A B}\left(P_{ \pm}+\epsilon \Gamma P_{\mp}\right) \eta=0 .
$$

Boundary conditions on the extrinsic curvature follow likewise from variation of the action. The boundary conditions are supersymmetric, in the sense that the full set of boundary conditions transforms into itself under supersymmetry transformations (i.e. each boundary condition is supersymmetric if the other boundary conditions are imposed). It is a remarkable fact that these are the only set of boundeary conditions which are consistent with anomaly cancellation and the unmodified supersymmetry transformations [26].

Supersymmetry transformations of the action are carried out subject to the gravitino and anti-symmetric tensor field boundary conditions. Without the $O\left(R^{2}\right)$ terms, the resulting action is supersymmetric to all orders in the parameter $\epsilon$, apart from one term which cancels the supersymmetry anomaly [17]. For the gravity anomaly to vanish it becomes necessary to add the higher-order curvature terms to the action, and then supersymmetry has only been established up to a limited order in the curvature [26]. 


\section{THE FIVE DIMENSIONAL REDUCTION}

In this section we shall present some of the details of a simple $E_{6} \times E_{8}$ reduction of the 11-dimensional theory to 5 dimensions. As we mentioned earlier, the bosonic sector for this reduction has been looked at in some detail in the old version of heterotic $M$-theory, especially by Lukas et al. 13. We shall give some new results for the topological and the fermionic terms in the action, but the main results are the set of boundary conditions for the 5-dimensional supergravity on a manifold with boundary.

The reduction uses a background gauge field on one boundary component $\partial \mathcal{M}_{1}$, related to the $S U(3)$ holonomy of the Calabi-Yau space used to compactify the internal dimensions. This is chosen because it induces a low-energy background $G$-flux due to the boundary condition (4).

The field content of the reduced theory is given in table II The single hypermultiplet includes the volume modulus $V$ along with a real and a complex scalar. This hypermultiplet has an $S U_{L}(2) \times S U_{R}(2)$ symmetry, with a $U(1)$ subgroup gauged by the graviphoton. (We shall use notation based on $S U_{L}(2) \times S U_{R}(2)$, rather than the equivalent description using $S U(2) \times S p(2)$.) The number of Abelian multiplets and the number of chiral matter multiplets are fixed by the Hodge numbers of the Calabi-Yau space. For this paper we shall only consider the $H^{1,1}$ moduli and leave the $H^{1,2}$ moduli for another occasion.

TABLE II: Field content of the reduced theory on the 5-dimensional manifold $\mathcal{M}$ with boundary components $\partial \mathcal{M}_{1}$ and $\partial \mathcal{M}_{2}$.

\begin{tabular}{llll}
\hline \hline Multiplet & Number & Location & Fields \\
\hline Graviton multiplet & 1 & $\mathcal{M}$ & $\left\{g_{\alpha \beta}, \mathcal{A}_{\alpha}, \psi_{\alpha}^{A}\right\}$ \\
Hypermultiplet & 1 & $\mathcal{M}$ & $\left\{V, \sigma, \xi, \zeta^{A}\right\}$ \\
Abelian multiplets & $h_{1,1}-1$ & $\mathcal{M}$ & $\left\{\mathcal{A}^{\perp i}, b^{i}, \lambda^{\perp A i}\right\}$ \\
$E_{6}$ Yang-Mills multiplet & 1 & $\partial \mathcal{M}_{1}$ & $\left\{A_{\mu}, \chi^{A}\right\}$ \\
$E_{6}$ Chiral matter multiplets & $h_{1,1}$ & $\partial \mathcal{M}_{1}$ & $\left\{C^{i p}\right\}$ \\
$E_{8}$ Yang-Mills multiplet & 1 & $\partial \mathcal{M}_{2}$ & $\left\{A_{\mu}, \chi^{A}\right\}$ \\
\hline \hline
\end{tabular}

The metric in 11 dimensions is chosen to reproduce the Einstein-Hilbert action for the metric in five-dimensions,

$$
d s^{2}=V^{-2 / 3} g_{\alpha \beta} d x^{\alpha} d x^{\beta}+V^{1 / 3}\left(g_{a \bar{b}} d x^{a} d x^{\bar{b}}+g_{\bar{a} b} d x^{\bar{a}} d x^{b}\right),
$$

where $g_{a \bar{b}}$ is the metric on a Calabi-Yau space of fixed volume $v$ and $V$ is the modulus field associated with changes to the Calabi-Yau volume. The background gauge field on the boundary $\partial \mathcal{M}_{1}$ induces the background $G$-flux in the bulk,

$$
G_{a b \bar{c} \bar{d}}=\sqrt{2} \alpha_{i} \nu_{a b \bar{c} \bar{d}}
$$

where the $\alpha_{i}$ are constants and $\nu_{a b \bar{c} \bar{d}}^{i}$ are generators of the Hodge-cohomology group $H^{2,2}$ of the Calabi-Yau space. Calabi-Yau moduli arise from expanding the Kähler form $\omega_{a \bar{b}}=i g_{a \bar{b}}$ in terms of the generators $\omega_{i a \bar{b}}$ of the Hodgecohomology group $H^{1,1}$

$$
\omega_{a \bar{b}}=b^{i} \omega_{i a \bar{b}}
$$

We use Hodge duality to choose $\omega_{i}=2 G_{i j} * \nu^{j}$, where $G_{i j}$ is the moduli space metric defined in Appendix $\mathrm{A}$ The moduli are constrained because the volume of $g_{a \bar{b}}$ is held constant,

$$
\mathcal{K}_{i j k} b^{i} b^{j} b^{k}=6 v
$$

where the tensor $\mathcal{K}_{i j k}$ is defined in Appendix A

The components of the antisymmetric tensor define additional scalar fields $\xi$ and vector fields $\mathcal{A}_{\alpha}^{i}$,

$$
\begin{aligned}
C_{a b c} & =\frac{i}{2} \xi \epsilon_{a b c} \\
C_{a \bar{b} \alpha} & =\sqrt{2} \mathcal{A}_{\alpha}{ }^{i} \omega_{i a \bar{b}}
\end{aligned}
$$

where $\epsilon_{a b c}$ is the covariantly constant 3-form on the Calabu-Yau space, normalised with

$$
\epsilon_{a b c} \epsilon^{a b c}=48 \text {. }
$$


The $C_{\alpha \beta \gamma}$ components define a scalar field $\sigma$ through dualisation (see below).

For the Fermi sector, we shall use the following set of $\Gamma$ matrices

$$
\begin{aligned}
\Gamma_{\alpha} & =V^{-1 / 3} \gamma_{\alpha} \otimes \gamma_{7}, \\
\Gamma_{a} & =V^{1 / 6} 1 \otimes \gamma_{a},
\end{aligned}
$$

where $\gamma_{\alpha}$ and $\gamma_{a}$ are sets of Dirac matrices in five and six dimensions respectively. These are chosen so that $\gamma_{\mu}^{*}=\gamma_{\mu}$ for $\mu=1 \ldots 4, \gamma_{5}^{*}=-\gamma_{5}$ and $\gamma_{7}^{*}=-\gamma_{7}$.

The Calabi-Yau space has two covariantly constant spinors $u_{A}$, with $\gamma_{7} u_{A}= \pm 1$ for $A=1,2$. We define $\bar{u}^{A}=\left(u_{A}\right)^{\dagger}$ and normalise so that

$$
\begin{aligned}
\bar{u}^{A} u_{B} & =\delta^{A}{ }_{B}, \\
\bar{u}^{A} \gamma_{7} u_{B} & =\tau^{A}{ }_{B},
\end{aligned}
$$

where $\tau$ is a diagonal matrix with $\tau^{1}{ }_{1}=-\tau^{2}{ }_{2}=1$.

The gravitino reduces to produce a gravitino $\psi_{\alpha}^{A}$ and superpartners $\zeta^{A}$ of the volume $V$ and $\lambda^{A i}$ of the moduli $b^{i}$,

$$
\begin{aligned}
& \Psi_{\alpha}=V^{-1 / 6}\left(\psi_{\alpha}^{A}-i \frac{\sqrt{3}}{2} \gamma_{\alpha} \zeta^{A}\right) \otimes u_{A}, \\
& \Psi_{a}=\frac{1}{2} V^{1 / 3} \lambda^{A i} \otimes \omega_{i a \bar{b}} \gamma^{\bar{b}} u_{A},
\end{aligned}
$$

where $\zeta^{A}=b_{i} \lambda^{A i} \sqrt{2}$. These particular combinations give the standard fermion kinetic terms in five dimensions.

On the boundary, we take the gauge field with the simplest non-trivial embedding which has a non-zero background $\tilde{\omega}_{a}$ on the boundary component $\partial \mathcal{M}_{1}$, breaking the $E_{8}$ symmetry down to $E_{6}$,

$$
\begin{aligned}
& A_{\mu}= \begin{cases}A_{\mu} \in \operatorname{ad}\left(E_{6}\right) & \text { on } \partial \mathcal{M}_{1}, \\
A_{\mu} \in \operatorname{ad}\left(E_{8}\right) & \text { on } \partial \mathcal{M}_{2},\end{cases} \\
& A_{a}= \begin{cases}\tilde{\omega}_{a}+\omega_{i a}{ }^{b} T_{b p} C^{i p} & \text { on } \partial \mathcal{M}_{1}, \\
0 & \text { on } \partial \mathcal{M}_{2},\end{cases}
\end{aligned}
$$

where the $T_{b p}$ are the $(3,27) E_{8}$ generators (see Appendix B).

The gaugino reduces to a four dimensional gaugino $\chi$ and the superpartner $\eta$ of the matter fields,

$$
\chi= \begin{cases}\chi^{A} \otimes u_{A}+\frac{1}{2} \omega_{i a}^{b} T_{b p} \eta^{A i p} \otimes \gamma^{a} u_{A}+\frac{1}{2} \omega_{i \bar{a} b} T^{b p} \eta^{A i}{ }_{p} \otimes \gamma^{\bar{a}} u_{A} & \text { on } \partial \mathcal{M}_{1}, \chi^{A} \in \operatorname{Ad}\left(E_{6}\right), \\ \chi^{A} \otimes u_{A} & \text { on } \partial \mathcal{M}_{2}, \chi^{A} \in \operatorname{Ad}\left(E_{8}\right) .\end{cases}
$$

We shall use the boundary conditions to confirm later that the $\mathrm{SU}(2)$ components of the fermion fields become chiral components on the boundary.

\section{A. Dualisation on a manifold with boundary}

During the reduction to five dimensions we replace the antisymmetric tensor $C_{\alpha \beta \gamma}$ with a scalar field $\sigma$ using a duality transformation. The subject of dualisation on a manifold with boundary is an interesting issue in its own right, and so we consider this subject in a slightly wider context below.

Consider a 5-dimensional action for a 4-form field strength tensor $G$ with Lagrangian

$$
\mathcal{L}_{0}(G)=A G_{\alpha \beta \gamma \delta} G^{\alpha \beta \gamma \delta}+B^{\alpha \beta \gamma \delta} G_{\alpha \beta \gamma \delta}+D,
$$

where $A, B$ and $D$ depend on other fields. This can be dualised by replacing the field strength $G$ with an arbitrary antisymmetric tensor $g$ and a scalar $\sigma$ with Lagrangian

$$
\mathcal{L}(g, \sigma)=A g_{\alpha \beta \gamma \delta} g^{\alpha \beta \gamma \delta}+B^{\alpha \beta \gamma \delta} g_{\alpha \beta \gamma \delta}+D-c \epsilon^{\alpha \beta \gamma \delta \epsilon} \sigma \partial_{\epsilon} g_{\alpha \beta \gamma \delta},
$$

where $c$ may depend on other fields. Variation with respect to $\sigma$ vanishes when the exterior derivative $d g$ of $g$ vanishes, and the field equations are equivalent to the ones obtained from $\mathcal{L}_{0}$. If the supersymmetry transformation of $g$ is 
chosen to be identical with the the supersymmetry transformations of $G$, then the Lagrangian $\mathcal{L}(g, \sigma)$ varies into $d g$ terms and the supersymmetry transformation of $\sigma$ can be chosen to make the action supersymmetric.

Now consider a boundary with a boundary condition on the field strength tensor,

$$
G_{\mu \nu \rho \sigma}=f_{\mu \nu \rho \sigma},
$$

where the indices are tangential to the boundary, and $f$ is some predetermined tensor, possibly depending on other fields. We can impose this boundary condition with a boundary action

$$
\mathcal{L}_{b}(g, \sigma)=-c \epsilon^{\mu \nu \rho \sigma}\left(g_{\mu \nu \rho \sigma}-f_{\mu \nu \rho \sigma}\right) \sigma .
$$

Supersymmetry of the action is assured as long as the boundary conditions, including this one, transform into one another under supersymmetry.

The dual theory is constructed by varying the action with respect to $g$, and then inserting the $g$ field equation into the action. This gives

$$
\mathcal{L}(\sigma)=\frac{6 c^{2}}{A}\left(\partial_{\epsilon} \sigma-\frac{1}{24 c} \epsilon_{\epsilon}^{\alpha \beta \gamma \delta} B_{\alpha \beta \gamma \delta}\right)^{2}+D .
$$

Part of the boundary term cancels an integration by parts, and we are left with,

$$
\mathcal{L}_{b}(\sigma)=c \epsilon^{\mu \nu \rho \sigma} f_{\mu \nu \rho \sigma} \sigma .
$$

Variation of the action now gives a boundary condition,

$$
\partial_{z} \sigma-\frac{1}{24 c} \epsilon^{\mu \nu \rho \sigma} B_{\mu \nu \rho \sigma}=\frac{A}{6 c} \epsilon^{\mu \nu \rho \sigma} f_{\mu \nu \rho \sigma}
$$

Furthermore, supersymmetry has been retained at each step, and so the boundary conditions of the dual theory transform into one another under supersymmetry.

In the case of heterotic $M$-theory, variation of $g$ in $\mathcal{L}(g, \sigma)$ gives the field equation

$$
g_{\alpha \beta \gamma \delta}=V^{-2} \epsilon_{\alpha \beta \gamma \delta}{ }^{\epsilon}\left(\partial_{\epsilon} \sigma-i\left(\xi \partial_{\epsilon} \bar{\xi}-\bar{\xi} \partial_{\epsilon} \xi\right)-\alpha_{i} \mathcal{A}^{i}+\text { fermi terms }\right) .
$$

The boundary condition on the field strength $G$ can be obtained from the exterior derivative of the boundary condition on $C$, Eq. (4). For the $S U(3) \times E_{6}$ reduction, the boundary has two components, $\partial \mathcal{M}_{1}$ with $E_{6}$ gauge fields, and $\partial \mathcal{M}_{2}$ with $E_{8}$ gauge fields. The boundary terms corresponding to Eq. (30) on $\partial \mathcal{M}_{2}$ resulting from the dualisation procedure are

$$
\mathcal{L}_{b}(\sigma)=-\frac{1}{8 \kappa_{5}^{2}} \epsilon \sigma \epsilon^{\mu \nu \rho \sigma} \operatorname{tr}\left(F_{\mu \nu} F_{\rho \sigma}\right)-\frac{1}{48 \kappa_{5}^{2}} \epsilon \sigma \epsilon^{\mu \nu \rho \sigma} \partial_{\mu}\left(V^{-1} \operatorname{tr} \bar{\chi} \gamma_{\nu \rho \sigma} \chi\right)
$$

The first term describes a coupling between the bulk $\sigma$ field and the Pontryagin density of the matter fields.

This is not quite the complete story for heterotic $M$-theory, because there are also boundary terms left over from the $C G G$ terms in the action when we integrate by parts to construct a Lagrangian $\mathcal{L}_{0}(G)$. These terms, which depend on $\mathcal{A}_{\mu}$ and $\xi$, combine with (33) to produce additional surface terms which have been absorbed into a covariant derivative term $\mathcal{D}_{\mu} \sigma$ in Eq. (C4) of Appendix C.

\section{B. Bulk action}

The contribution to the action from the bulk is given by

$$
S_{S G}=\frac{1}{2 \kappa_{5}^{2}} \int_{\mathcal{M}} \mathcal{L}_{S G}
$$

where $\kappa_{5}^{2}=\kappa_{11}^{2} / v$. The five-dimensional Lagrangian was obtained by Lukas et al. by a combination of the reduction ansatz for the bosonic terms and then by a comparison with the known five-dimensional supergravity models. We have checked most of the two-fermion terms directly using the reduction ansatz given above. Compared to earlier work, we find differences with some of the mass terms. There is an independent consistency check on the mass terms 
from requiring that the vacuum energy vanishes. The vacuum energy was evaluated in [29], and this consistency check is satisfied by the new Lagrangian, but not by the old one.

The Lagrangian with up to two fermion fields is given in Appendix C. We use the covariant $\sigma$ derivative based upon Eq. (??),

$$
\mathcal{D}_{\alpha} \sigma=\partial_{\alpha} \sigma-i\left(\xi \partial_{\alpha} \bar{\xi}-\bar{\xi} \partial_{\alpha} \xi\right)-\alpha_{i} \mathcal{A}_{\alpha}^{i}
$$

Cross-terms between the derivatives of the hypermultiplet scalars and fermions have been absorbed into the fermion derivatives,

$$
\begin{aligned}
\mathcal{D}_{\alpha} \psi_{\beta}^{A} & =\left(\nabla_{\alpha}+\mathcal{A}_{\alpha}^{i} \mathcal{P}_{i}\right) \psi_{\beta}^{A}+\omega_{L B \alpha}^{A} \psi_{\beta}^{B}, \\
\mathcal{D}_{\alpha} \lambda^{A i} & =\left(\nabla_{\alpha}+\mathcal{A}_{\alpha}^{i} \mathcal{P}_{i}\right) \lambda^{A i}+\partial_{\alpha} b^{j} \Gamma^{i}{ }_{j k} \lambda^{A k}+\omega_{L B \alpha}^{A} \lambda^{B}, \\
\mathcal{D}_{\alpha} \zeta^{A} & =\left(\nabla_{\alpha}+\mathcal{A}_{\alpha}^{i} \mathcal{P}_{i}\right) \zeta^{A}+\omega_{R B}^{A} \zeta^{B},
\end{aligned}
$$

where $\mathcal{P}_{i}=-\frac{1}{4} i V^{-1} \alpha_{i} \tau$, and the hypermultiplet $S U(2)$ connections are

$$
\omega_{L B \alpha}^{A}=\frac{1}{4}\left(\begin{array}{cc}
i V^{-1} \mathcal{D}_{\alpha} \sigma & -4 V^{-1 / 2} \partial_{\alpha} \xi \\
4 V^{-1 / 2} \partial_{\alpha} \bar{\xi} & -i V^{-1} \mathcal{D}_{\alpha} \sigma
\end{array}\right), \quad \omega_{R B \alpha}^{A}=\frac{3}{4}\left(\begin{array}{cc}
-i V^{-1} \mathcal{D}_{\alpha} \sigma & 0 \\
0 & i V^{-1} \mathcal{D}_{\alpha} \sigma
\end{array}\right) .
$$

These connections are associated with the hypermultiplet tetrad

$$
E_{B \alpha}^{A}=\left(\begin{array}{cc}
V^{-1}\left(\partial_{\alpha} V-i \mathcal{D}_{\alpha} \sigma\right) & 2 V^{-1 / 2} \partial_{\alpha} \xi \\
-2 V^{-1 / 2} \partial_{\alpha} \bar{\xi} & V^{-1}\left(\partial_{\alpha} V+i \mathcal{D}_{\alpha} \sigma\right)
\end{array}\right)
$$

The tetrad is defined so that the hypermultiplet kinetic terms can be written as an $S U(2) \operatorname{trace,} \operatorname{tr}\left(E_{\alpha} E^{\dagger \alpha}\right) / 4$.

\section{Boundary action}

The first surface terms we consider are ones obtained from a reduction of the the supergravity boundary terms (2),

$$
S_{0}=\frac{1}{\kappa_{5}^{2}} \int_{\partial \mathcal{M}_{1}} \mathcal{L}_{0}\left(\partial \mathcal{M}_{1}\right)+\frac{1}{\kappa_{5}^{2}} \int_{\partial \mathcal{M}_{2}} \mathcal{L}_{0}\left(\partial \mathcal{M}_{2}\right)
$$

The Lagrangians are

$$
\begin{aligned}
& \mathcal{L}_{0}\left(\partial \mathcal{M}_{1}\right)=K-\frac{\sqrt{2}}{2} V^{-1} \alpha-\frac{1}{4} \tau^{A}{ }_{B} \bar{\psi}_{A \mu} \gamma^{\mu \nu} \bar{\psi}_{\nu}^{B}-\frac{1}{4} \tau^{A}{ }_{B} \bar{\zeta}_{A} \zeta^{B}-\frac{1}{4} \tau^{A}{ }_{B} G_{i j}^{\perp} \bar{\lambda}_{A}^{i} \lambda^{B j}+\mathcal{T}, \\
& \mathcal{L}_{0}\left(\partial \mathcal{M}_{2}\right)=K+\frac{\sqrt{2}}{2} V^{-1} \alpha+\frac{1}{4} \tau^{A}{ }_{B} \bar{\psi}_{A \mu} \gamma^{\mu \nu} \bar{\psi}_{\nu}^{B}+\frac{1}{4} \tau^{A}{ }_{B} \bar{\zeta}_{A} \zeta^{B}+\frac{1}{4} \tau^{A}{ }_{B} G_{i j}^{\perp} \bar{\lambda}_{A}^{i} \lambda^{B j}+\mathcal{T},
\end{aligned}
$$

where $\mathcal{T}$ is a fermionic torsion term which depends on gravitino components normal to the boundary. The torsion term cancels a total derivative of the torsion in $R(\Omega)$, and never appears in the field equations. These boundary Lagrangians are consistent with the $\zeta=\lambda=0$ case in [21].

The remaining surface terms contain the matter fields and their couplings to the bulk supergravity fields,

$$
S_{Y M}=\frac{1}{g^{2}} \int_{\partial \mathcal{M}_{1}} \mathcal{L}_{Y M}\left(\partial \mathcal{M}_{1}\right)+\frac{1}{g^{2}} \int_{\partial \mathcal{M}_{2}} \mathcal{L}_{Y M}\left(\partial \mathcal{M}_{2}\right)
$$

where $g^{2}=\kappa_{11}^{2} /(\epsilon v)$. The Lagrangian for the $E_{8}$ Yang-Mills multiplet is

$$
\mathcal{L}_{Y M}\left(\partial \mathcal{M}_{2}\right)=-\frac{1}{4} V F_{\mu \nu}^{I} F^{I \mu \nu}-\frac{1}{2} \bar{\chi}_{A}^{I} \gamma^{\mu} D_{\mu} \chi^{I A}-\bar{\psi}_{A \mu} j^{A \mu}-\bar{\zeta}_{A} j^{A}+\Theta^{\mu} \mathcal{D}_{\mu} \sigma
$$

where $I$ labels the $E_{8}$ Lie algebra basis. The bulk fermions couple to fermionic currents $j^{A \mu}$, $j^{A}$, and $\sigma$ to the topological current $\Theta^{\mu}$ given by

$$
\begin{aligned}
j^{A \mu} & =\frac{1}{4} V^{1 / 2} \gamma^{\rho \sigma} \gamma^{\mu} F_{\rho \sigma}^{I} \chi^{A I} \\
j^{A} & =i \frac{\sqrt{2}}{4} V^{1 / 2} \gamma^{\rho \sigma} F_{\rho \sigma}^{I} \chi^{A I} \\
\Theta^{\mu} & =\frac{1}{12} \epsilon^{\mu \nu \rho \sigma}\left(\omega_{\nu \rho \sigma}^{Y}+\frac{1}{4} V^{-1} \bar{\chi}_{A}^{I} \gamma_{\nu \rho \sigma} \chi^{A I}\right)
\end{aligned}
$$


Note that $j^{A \mu}$ is the usual supercurrent for the gauge multiplet.

The Lagrangian for the $E_{6}$ Yang-Mills multiplet and the matter terms is given in Appendix C. Part of the potential depends on the $D$-term,

$$
D^{I}=2 \bar{C}^{i} \Lambda^{I} C_{i}
$$

The remaining part of the potential depends on the superpotential $W$, which can be determined most simply from examination of the $\eta$-mass terms,

$$
W=\frac{2 \sqrt{3}}{3} \mathcal{K}^{-1} \mathcal{K}_{i j k} d_{p q r} C^{i p} C^{j q} C^{k r}
$$

The coupling to the bulk fields again depends on the supercurrent, now given in Eqs. (C5, C8). There are also bulk field contributions to the derivatives,

$$
\mathcal{D}_{\mu} C^{i p}=D_{\mu} C^{i p}+\partial_{\mu} b^{k} \Gamma^{i}{ }_{j k} C^{j p}
$$

where the moduli-space connection coefficients are given in Eq. (A14).

\section{Boundary conditions}

The boundary conditions for the 5-dimensional theory can be obtained by reducing the boundary conditions Eqs. (4) and (6), or by variation of the full reduced action, including the boundary terms. The fermionic boundary conditions in five dimensions are expressed in terms of chiral projection operators,

$$
P_{+}{ }_{B}{ }_{B}=\left(\begin{array}{cc}
P_{L} & 0 \\
0 & P_{R}
\end{array}\right), \quad P_{-}{ }_{B}{ }_{B}=\left(\begin{array}{cc}
P_{R} & 0 \\
0 & P_{L}
\end{array}\right),
$$

where $P_{L}=\frac{1}{2}\left(1+\gamma_{5}\right)$ and $P_{R}=\frac{1}{2}\left(1-\gamma_{5}\right)$. In order to facilitate reduction to four dimensions, we take $\gamma_{5}$ to be the $\gamma$-matrix component in the direction of the ingoing unit normal on the $E_{6}$ boundary component $\partial \mathcal{M}_{1}$ and along the outgoing unit normal on the $E_{8}$ boundary component $\partial \mathcal{M}_{2}$. We also use $\mathcal{D}_{5}$ to denote the derivative along the $i n w a r d$ unit normal on the $E_{6}$ boundary, and the outward unit normal on the $E_{8}$ boundary, but for the extrinsic curvature we always use the outgoing normals.

If we drop the 3 -fermi terms, then the fermion boundary conditions on the bulk fields become

$$
\begin{aligned}
P_{-}{ }_{B}{ }_{B} \psi_{\mu}^{B} & =\epsilon\left(\delta_{\mu}{ }^{\nu}-\frac{1}{4} \gamma_{\mu} \gamma^{\nu}\right) \tau^{A}{ }_{B} j_{\nu}^{B}, \\
P_{+}{ }^{A}{ }_{B} \lambda^{B i} & =-\epsilon \tau^{A}{ }_{B} j^{B i}, \\
P_{+}{ }^{A}{ }_{B} \zeta^{B} & =-\epsilon \tau^{A}{ }_{B} j^{B},
\end{aligned}
$$

where $\epsilon=\kappa_{5}^{2} / g^{2}$. The fermionic currents on the right-hand side of these equations are given in Eqs. (C5 C7) for the $E_{6}$ boundary component, and in Eqs. 46,47) for the $E_{8}$ boundary component. For the matter fields,

$$
P_{-}{ }_{B} \chi^{I B}=P_{+}{ }^{A}{ }_{B} \eta^{B i p}=0
$$

These enable us to identify the chiral components of the Majorana spinors as

$$
\begin{array}{cc}
\chi_{L}{ }^{I} \equiv \chi^{1 I}, & \chi_{R}{ }^{I} \equiv-\chi^{2 I} \\
\eta_{L}{ }^{i p} \equiv \eta^{2 i p}, & \eta_{R}{ }^{i p} \equiv \eta^{1 i p}
\end{array}
$$

The minus sign appears in $\chi_{R}$ to ensure that $\chi \equiv \chi_{R}+\chi_{L}$ is real. Chirality is defined for a conjugate spinor $\bar{\chi}$ by $\bar{\chi}_{L}=\bar{\chi} P_{L}$, so we have $\bar{\chi}_{L}=\overline{\chi_{R}}$.

The boundary conditions on the bosonic fields which result from a reduction of the boundary condition (4) on the $E_{8}$ component $\partial \mathcal{M}_{2}$ are

$$
\begin{aligned}
\xi & =-\frac{1}{2} \epsilon V^{1 / 2} \bar{\chi}_{L}{ }^{I} \chi_{L}{ }^{I} \\
\mathcal{A}_{\mu}^{i} & =\frac{i}{4} \epsilon \tau^{A}{ }_{B} b^{i} \bar{\chi}_{A}{ }^{I} \gamma_{\mu} \chi^{B I},
\end{aligned}
$$


where the moduli-space tensors are defined in Appendix @. These boundary conditions raise a problem with the supersymmetry transformation of the $\mathcal{A F F}$ term in the action, which gives a boundary term involving $\mathcal{A}$. In eleven dimensions, this is the term which cancels with the supersymmetry anomaly. In five dimensions, it seems that we have to live with this variation since we have lost the quantum anomaly when we threw out the high-energy modes. This variation is $O\left(\epsilon^{3}\right)$, and since the string corrections in heterotic string theory appear at $O(\epsilon)$, we might regard the $O\left(\epsilon^{3}\right)$ variation as a 'small' correction. (In related work on five-dimensional supergravity, Ref. 21] imposes $\mathcal{A}_{\mu}^{i}=0$, but that paper includes distributions, which we seek to avoid.)

The boundary condition for $\sigma$ on $\partial \mathcal{M}_{2}$ is most easily obtained by the variation of $\sigma$ in the full action, including the surface terms with Lagrangian (45),

$$
\begin{aligned}
\mathcal{D}_{5} \sigma= & -2 \epsilon V^{2} \partial_{\mu} \Theta^{\mu}+\frac{3}{4} i V \tau_{B}^{A} \bar{\zeta}_{A} \gamma_{5} \zeta^{B}-\frac{1}{4} i V \tau^{A}{ }_{B} G_{i j}^{\perp} \bar{\lambda}_{A}^{i} \gamma_{5} \lambda^{B j} \\
& +\frac{1}{4} i V \tau^{A}{ }_{B} \bar{\psi}_{A \alpha} \gamma^{\alpha \beta} \gamma_{5} \psi_{\beta}^{B}-\frac{\sqrt{2}}{2} V \tau^{A}{ }_{B} \bar{\zeta}_{A} \gamma^{\alpha} \gamma_{5} \psi_{\alpha}^{B},
\end{aligned}
$$

where $\Theta^{\mu}$ is given by Eq. (48). From the variation of $V$,

$$
\partial_{5} V=-\sqrt{2} \alpha+2 \epsilon V^{2} \frac{\partial \mathcal{L}_{Y M}}{\partial V}+i \frac{\sqrt{2}}{2} V \bar{\zeta}_{A} \gamma^{\alpha} \gamma_{5} \psi_{\alpha}^{A} .
$$

The boundary conditions on the extrinsic curvature can be obtained by variation of the metric,

$$
\begin{aligned}
K^{\mu \nu}-K g^{\mu \nu}= & \kappa_{5}^{2} T_{Y M}^{\mu \nu}+\frac{\sqrt{2}}{2} \alpha V^{-1} g^{\mu \nu}+\frac{1}{2} \tau^{A}{ }_{B} \bar{\psi}_{A \rho} \gamma^{\mu \rho} \psi^{B \nu}, \\
& +\frac{1}{4} \tau^{A}{ }_{B}\left(\bar{\psi}_{A \rho} \gamma^{\rho \sigma} \psi_{\sigma}^{B}+\bar{\zeta}_{A} \zeta^{B}+G_{i j}^{\perp} \bar{\lambda}_{A}^{i} \lambda^{B j}\right) g^{\mu \nu},
\end{aligned}
$$

where the surface stress tensor for the matter on $\partial \mathcal{M}_{2}$ is

$$
T_{Y M}^{\mu \nu}=\frac{1}{g^{2}}\left(2 \frac{\delta \mathcal{L}_{Y M}}{\delta g_{\mu \nu}}-g^{\mu \nu} \mathcal{L}_{Y M}\right)
$$

The bulk-fermion bilinear terms in these bosonic boundary conditions can be re-written in a variety of ways using the fermion boundary conditions.

On the $E_{6}$ boundary component $\partial \mathcal{M}_{1}$ we use the Lagrangians given in Appendix (C),

$$
\begin{aligned}
\xi= & \frac{1}{2} \epsilon V^{1 / 2} \bar{\chi}_{L}{ }^{I} \chi_{L}{ }^{I}-\frac{\sqrt{3}}{3} \epsilon \epsilon_{a b c} d_{p q r} \mathcal{K}^{-1} \mathcal{K}_{i j k} C^{i p} C^{j q} C^{k r} \\
\mathcal{A}_{\mu}^{i}= & -\frac{i}{4} \epsilon \tau^{A}{ }_{B} b^{i} \bar{\chi}_{A}{ }^{I} \gamma_{\mu} \chi^{B I}-i \epsilon \Gamma_{j k}^{i}\left(C^{j p} \mathcal{D}_{\mu} \bar{C}^{k}{ }_{p}-\bar{C}^{k}{ }_{p} \mathcal{D}_{\mu} C^{j p}\right), \\
& +\frac{i}{4} \epsilon\left(\Gamma^{i}{ }_{j k}-b^{i} G_{j k}\right)\left(\bar{\eta}_{R}^{j} \gamma_{\mu} \eta_{L}^{k p}-\bar{\eta}_{L}^{j p} \gamma_{\mu} \eta_{R p}^{k}\right) \\
\mathcal{D}_{5} \sigma= & -2 \epsilon V^{2} \partial_{\mu} \Theta^{\mu}+\frac{3}{4} i V \tau^{A}{ }_{B} \bar{\zeta}_{A} \gamma_{5} \zeta^{B}-\frac{1}{4} i V \tau^{A}{ }_{B} G_{i j}^{\perp} \bar{\lambda}_{A}^{i} \gamma_{5} \lambda^{B j} \\
& +\frac{1}{4} i V \tau^{A}{ }_{B} \bar{\psi}_{A \alpha} \gamma^{\alpha \beta} \gamma_{5} \psi_{\beta}^{B}-\frac{\sqrt{2}}{2} V \tau^{A}{ }_{B} \bar{\zeta}_{A} \gamma^{\alpha} \gamma_{5} \psi_{\alpha}^{B} \\
K^{\mu \nu}-K g^{\mu \nu}= & -\sqrt{2} \alpha-2 \epsilon V^{2} \frac{\partial \mathcal{L}_{Y M}}{\partial V}+i \frac{\sqrt{2}}{2} V \bar{\zeta}_{A} \gamma^{\mu \nu} \gamma^{\alpha} \gamma_{5} \psi_{\alpha}^{A}, \\
& -\frac{1}{4} \tau^{A}{ }_{B}\left(\bar{\psi}_{A \rho} \gamma^{\rho \sigma} \psi_{\sigma}^{B}+\bar{\zeta}_{A} \zeta^{B}+G^{B \nu}-\frac{1}{2} \tau^{A}{ }_{B} \bar{\psi}_{A \rho} \gamma^{\mu \rho} \bar{\lambda}_{A}{ }^{\mu} \lambda^{B j}\right) \psi^{B \nu}
\end{aligned}
$$

Note that the boundary conditions have a simple solution where all of the fields vanish apart from $V=1-\sqrt{2} \alpha x^{5}$ and $g_{\mu \nu}=V^{1 / 3} \eta_{\mu \nu}$, where $\eta_{\mu \nu}$ is the flat Minkowski metric. These also satisfy the field equations, and form the background for a reduction of the theory to four dimensions. 


\section{E. Gaugino condensates}

An important question we have to address is the extent to which a gaugino condensate on either boundary can affect the boundary conditions, and whether the condensate can break the supersymmetry as a consequence. The direct effect of a gaugino condensate on the fermion boundary conditions is a special feature of the corrected heterotic $M$-theory, where it appears in the chiral projection as the term $\Gamma$ in (6). The consequences for the gravitino in five dimensions have already been addressed in Ref. [9], where the Calabi-Yau space had a topology with Hodge number $h_{1,1}=1$. We are now in a position to give the modified boundary conditions for the general case.

The reduction continues as before, but this time we introduce the gaugino condensate on the boundary component $\partial \mathcal{M}_{n}$

$$
\left\langle\bar{\chi}^{I} \Gamma_{a b c} \chi^{I}\right\rangle=\Lambda_{n} \epsilon_{a b c},
$$

where $\Lambda_{n} \equiv \Lambda_{n}(V)$. Other boundary fermion fields will be set to zero. The condensate appears in the boundary condition for the antisymmetric tensor (4), producing an affect on the boundary conditions for the field $\xi$ in five dimensions,

$$
\xi= \begin{cases}\frac{1}{2} i \epsilon \Lambda_{1}-\frac{\sqrt{3}}{3} \epsilon \epsilon_{a b c} d_{p q r} \mathcal{K}^{-1} \mathcal{K}_{i j k} C^{i p} C^{j q} C^{k r} & \text { on } \partial \mathcal{M}_{1}, \\ -\frac{1}{2} i \epsilon \Lambda_{2} & \text { on } \partial \mathcal{M}_{2} .\end{cases}
$$

These boundary conditions imply that the field $\xi$ develops a vacuum expectation value determined by $\Lambda_{1}$ and $\Lambda_{2}$ [19]. This is equivalent to generating a background $G$-flux (see Eq. (13)), and it is this mechanism in heterotic $M$-theory which produces the condensate-induced superpotential $W_{g}$ in four dimensions. The four-dimensional supersymmetry is broken, except for special values of $V$ where the super-derivatives of $W_{g}$ vanish and the four dimensional supersymmetry is restored. There are no other contributions from the fermion condensate to the bosonic boundary conditions.

The fermion boundary conditions (6) include the $\Gamma$-correction to the chiral projection operator,

$$
\begin{aligned}
\left(P_{-}+\Gamma_{n} P_{+}\right){ }_{B}^{A} \psi_{\mu}^{B} & =0, \\
P_{+}{ }^{A}{ }_{B} \lambda^{B i} & =0 \\
P_{+}{ }^{A}{ }_{B} \zeta^{B} & =0
\end{aligned}
$$

where

$$
\Gamma_{n}=\frac{1}{2} \epsilon \Lambda_{n} V^{-1 / 2}\left(\begin{array}{ll}
0 & 1 \\
1 & 0
\end{array}\right) .
$$

The gravitino boundary condition can be regarded as a chiral twist between the two boundaries. The result given here agrees with Ref. [9]. The gravitino boundary condition breaks the five-dimensional supersymmetry via quantum effects, specifically by producing a non-zero vacuum energy. This vacuum energy was calculated in Ref. [9]. There are no condensate contributions to the other fermion boundary conditions. Note that there are contributions from the condensate-induced $G$-flux to the fermion mass terms, but these are related to the superpotential and do not contribute to the vacuum energy. (The fermion mass terms were used to calculate the superpotential in Ref. [19].)

\section{CONCLUSION}

We have completed the rather modest task of reducing the simplest version of heterotic $M$-theory using a CalabiYau three-fold to produce a new supergravity model on a manifold with boundary in five dimensions. Many features of the the theory are familiar from the old literature [13, 14]. The new results are the in the boundary action and the boundary conditions on the bulk fields. These contain features which are worthy of further investigation.

One example is that the Pontryagin density of the Yang-Mills gauge fields appears in the boundary condition for the hypermultiplet scalar $\sigma$. The appearance of topological terms here in the boundary conditions has its origin in the dualisation of the Abelian gauge field on the manifold with boundary (see Sect. IIIA), and is the origin of the Pontryagin density terms in the four-dimensional action. It is possible that this hypermultiplet scalar plays a role in gaugino condensation, since gaugino condensation is associated with instanton effects. 
The principal motivation for the reduction has been to incorporate gaugino condensation effects into the boundary conditions, because it has been argued that these can provide an uplifting mechanism for the potential in the context of moduli stabilisation. The results here are quite simple: the gravitino boundary conditions give a twist in the chirality of the gravitino which is proportional to the magnitude of the gaugino condensate. The only effect of the gaugino condensate on the bosonic sector is to source a background $G$-flux, which contributes to the moduli fields' superpotential.

The consequences of twisting the fermion boundary conditions on the vacuum energy have been related elsewhere 9] but, so far, no-one has analysed the effects on such 1-loop quantum calculations of the coupling between the bulk and boundary modes present in the boundary conditions (53.55). This coupling may also be relevant to the calculation of anomalies on a manifold with boundary.

The present work has been restricted to the $H^{1,1}$ moduli of the Calabi-Yau space. We hope to be able to complete a similar analysis for the $H^{2,1}$ moduli if there is sufficient interest. More ambitious yet would be to consider other reductions of heterotic $M$-theory which are more relevant to particle phenomenology [5]. The main problem here is the inclusion five-branes, which are required for anomaly cancellation. A consistent approach, like the one we have adopted, should include the back-reaction of the matter and curvature of the the five-brane, but this is not possible with the present understanding of the five-brane. However, if we ignore the content of the five-brane, it should be possible to combine boundary conditions on the bulk fields at the boundaries with junction conditions across the five-branes.

\section{Acknowledgments}

John Omotani and Paul Saffin receive support from the Science and Technology Facilities Council.

\section{Appendix A: Calabi-Yau moduli spaces}

Some of the definitions and results concerning the moduli spaces of metric deformations of a Calabi-Yau space (based on Ref. [30]) used in the body of the paper have been collected together in this appendix. The Kähler form $\omega_{a \bar{b}}$ and the metric tensor $g_{a \bar{b}}$ on the Calabi-Yau space are related by

$$
\omega_{a \bar{b}}=i g_{a \bar{b}}
$$

The volume is given by

$$
v=\frac{1}{6} \int \omega \wedge \omega \wedge \omega .
$$

We choose a fixed set of generators of the cohomology group $H^{1,1}$ and denote these by $\omega_{i a \bar{b}}$. The Kähler form can be expanded using real moduli fields $b^{i}$,

$$
\omega_{a \bar{b}}=b^{i} \omega_{i a \bar{b}}
$$

Two important tensors on the moduli space are the metric

$$
G_{i j}=\frac{1}{2 v} \int \omega_{i} \wedge * \omega_{j},
$$

and the intersection tensor,

$$
\mathcal{K}_{i j k}=\int \omega_{i} \wedge \omega_{j} \wedge \omega_{k}
$$

The metric and its inverse can be used to raise and lower indices in the usual way. We also use the notation,

$$
\mathcal{K}=\mathcal{K}_{i j k} b^{i} b^{j} b^{k}=6 v
$$

Useful identities which follow from Eqs. (A2) and (A4) are

$$
* \omega_{i}=b_{i} \omega \wedge \omega-\omega \wedge \omega_{i}
$$


and

$$
G_{i j}=-\frac{1}{2} \omega_{i a}{ }^{b} \omega_{j b}{ }^{a}
$$

The first identity can be used with (A4) and (A5) to obtain another series of useful formulae,

$$
\begin{aligned}
\mathcal{K}^{-1} \mathcal{K}_{i j k} b^{k} & =\frac{2}{3} b_{i} b_{j}-\frac{1}{3} G_{i j}, \\
\mathcal{K}^{-1} \mathcal{K}_{i j k} b^{j} b^{k} & =\frac{2}{3} b_{i}, \quad b_{i} b^{i}=\frac{3}{2} .
\end{aligned}
$$

These imply that

$$
G_{i j}=-\frac{1}{2} \frac{\partial \ln \mathcal{K}}{\partial b^{i} \partial b^{j}}
$$

The tangent space to the moduli space can be decomposed into the direction along $b^{i}$ and the perpendicular direction by using a projection tensor,

$$
\delta^{\perp}{ }_{i}{ }^{j}=\delta_{i}{ }^{j}-\frac{2}{3} b_{i} b^{j}
$$

Tensor components orthogonal to $b^{i}$ will generally be denoted by the superscript $\perp$.

By differentiating (A8) with respect to $b^{i}$ we obtain a set of metric-connection coefficients,

$$
\Gamma_{i j k}=-\frac{i}{2} \omega_{i a}^{b} \omega_{j b}^{c} \omega_{k c}^{a}
$$

The Levi-Civita connection components follow from differentiating (A11),

$$
\Gamma_{i(j k)}=-\frac{3}{2} \mathcal{K}^{-1} \mathcal{K}_{i j k}-3 b_{(i} G^{\perp}{ }_{j k)}
$$

The corresponding curvature components of the Levi-Civita connection are

$$
R_{i j k l}=\Gamma_{m(i l)} \Gamma_{(j k)}^{m}-\Gamma_{m(i k)} \Gamma_{(j l)}^{m},
$$

which work out as

$$
R_{i j k l}=\frac{9}{4} \mathcal{K}^{-2} \mathcal{K}_{i l}{ }^{m} \mathcal{K}_{k j m}-\frac{9}{4} \mathcal{K}^{-2} \mathcal{K}_{i k}{ }^{m} \mathcal{K}_{l j m}+\frac{1}{2} G_{i l} G_{k j}-\frac{1}{2} G_{i k} G_{l j}
$$

Finally, we collect together the combinations of the cohomology generators which arise frequently in the dimensional reduction of the action,

$$
\begin{aligned}
\omega_{i a}{ }^{a} & =2 i b_{i} \\
\omega_{i a}{ }^{b} \omega_{j b}{ }^{a} & =-2 G_{i j}, \\
\omega_{i a}{ }^{b} \omega_{j b}{ }^{c} & =-i \Gamma^{k}{ }_{i j} \omega_{k a}{ }^{c}, \\
\omega_{i[a}{ }^{c} \omega_{j b]}{ }^{d]} & =-\frac{3}{4} i \mathcal{K}^{-1} \mathcal{K}_{i j}{ }^{k} \varepsilon_{a b e} \varepsilon^{c d f} \omega_{k}{ }^{e} f \\
\omega_{i a}{ }^{[d} \omega_{j b}{ }^{e} \omega_{k c}{ }^{f]} & =-\frac{1}{48} i \mathcal{K}^{-1} \mathcal{K}_{i j k} \varepsilon_{a b c} \varepsilon^{d e f}, \\
\omega_{i a}{ }^{b} \omega_{j b}{ }^{c} \omega_{k c}{ }^{d} \omega_{l d}{ }^{a} & =2 \Gamma^{m}{ }_{i j} \Gamma_{m k l} .
\end{aligned}
$$

\section{Appendix B: $E_{8}$ group theory identities}

This appendix contains the identities used to reduce the $E_{8}$ group theory down to its $\operatorname{subgroup~} S U(3) \times E_{6}$. Although this work is standard material, it is included to make plain our choices for the normalisation factors and signs. We start from reduction of the adjoint representation of $E_{8}$ into $S U(3) \times E_{6}$ representations,

$$
248=(8,1) \oplus(1,78) \oplus(3,27) \oplus(\overline{3}, \overline{2} \overline{7}) .
$$


The corresponding $E_{8}$ generators are

$$
S^{i}, X^{I}, T_{a p}, T^{a p},
$$

where the $S^{i}$ generate the $\mathrm{SU}(3)$ subgroup, the $X^{I}$ generate the $E_{6}$ subgroup, and $\left(T_{a p}\right)^{\dagger}=T^{a p}$ generate the cosets. We denote the generators of $S U(3)$ in the fundamental representation by $\left(\lambda^{i}\right)_{a}^{b}$, and the generators of $E_{6}$ in the fundamental representation by $\left(\Lambda^{I}\right)_{p}{ }^{q}$. (The $\mathrm{SU}(3)$ indices $a$ and $b$ used in this appendix are raised and lowered using complex conjugation, not with the Calabi-Yau metric).

The generators are normalised so that

$$
\begin{aligned}
\operatorname{Tr}\left(S^{i} S^{j}\right) & =30 \delta^{i j}, \\
\operatorname{Tr}\left(X^{I} X^{J}\right) & =30 \delta^{I J}, \\
\operatorname{Tr}\left(T^{a p} T_{b q}\right) & =\delta_{b}^{a} \delta_{q}^{p}, \\
\operatorname{Tr}\left(\lambda^{i} \lambda^{j}\right) & =\frac{1}{2} \delta^{i j}, \\
\operatorname{Tr}\left(\Lambda^{I} \Lambda^{J}\right) & =3 \delta^{I J} .
\end{aligned}
$$

This hotchpotch of normalisation constants turns out to be convenient.

We require the $T_{a p}$ to transform as the fundamental of $\mathrm{SU}(3)$ and the fundamental of $E_{6}$, so we find

$$
\begin{aligned}
{\left[S^{i}, T_{a p}\right] } & =\lambda_{a}^{i}{ }^{b} T_{b p}, \\
{\left[X^{I}, T_{a p}\right] } & =\Lambda_{p}^{I}{ }_{p}^{q} T_{a q} .
\end{aligned}
$$

For the remaining commutators,

$$
\begin{aligned}
{\left[T_{a p}, T_{b q}\right] } & =\frac{1}{\sqrt{6}} \hat{\epsilon}_{a b c} d_{p q r} T^{c r}, \\
{\left[T_{a p}, T^{b q}\right] } & =-\frac{1}{30} \delta_{a}{ }^{b} \Lambda_{p}^{I}{ }^{q} X^{I}-\frac{1}{30} \delta_{p}{ }^{q} \lambda_{a}^{i}{ }_{a}^{b} S^{i},
\end{aligned}
$$

where $\hat{\epsilon}_{a b c}$ is the Levi-Civita tensor and $d_{p q r}$ is an $E_{6}$ symmetric tensor, normalised by

$$
\hat{\epsilon}_{a b c} \hat{\epsilon}^{a b c}=6, \quad d_{p r s} d^{q r s}=\delta_{p}^{q} .
$$

The trace of a triple product becomes

$$
\operatorname{Tr}\left(T_{a p} T_{b q} T_{c r}\right)=\frac{1}{\sqrt{24}} \hat{\epsilon}_{a b c} d_{p q r}
$$

Finally, outer products of the $S U(3)$ and $E_{6}$ generators are given by

$$
\begin{aligned}
\left(\lambda^{i}\right)_{c}^{a}\left(\lambda^{i}\right)_{d}^{b} & =\frac{1}{2}\left(\delta_{d}^{a} \delta_{c}^{b}-\frac{1}{3} \delta_{c}^{a} \delta_{d}^{b}\right) \\
\left(\Lambda^{I}\right)_{q}^{p}\left(\Lambda^{I}\right)_{s}^{r} & =\frac{1}{6}\left(\delta_{q}^{p} \delta_{s}^{r}+3 \delta_{s}^{p} \delta_{q}^{r}-30 d_{q s t} d^{p r t}\right) .
\end{aligned}
$$




\section{Appendix C: Lagrangians}

The bulk Lagrangian for the $E_{6} \times E_{8}$ reduction of heterotic $M$-theory, when truncated at the fermion bilinear terms, is given by,

$$
\begin{aligned}
\mathcal{L}_{S G}= & -R(\Omega)-\frac{1}{2} V^{-2} \partial^{\alpha} V \partial_{\alpha} V-\frac{1}{2} V^{-2} \mathcal{D}_{\alpha} \sigma \mathcal{D}^{\alpha} \sigma-2 V^{-1} \partial^{\alpha} \bar{\xi} \partial_{\alpha} \xi-G^{\perp}{ }_{i j} \partial_{\alpha} b^{i} \partial^{\alpha} b^{j} \\
& -\frac{1}{4} \mathcal{F}_{i \alpha \beta} \mathcal{F}^{i \alpha \beta}-\mathcal{K}^{-1} \mathcal{K}_{i j k} \epsilon^{\alpha \beta \gamma \delta \epsilon} \mathcal{A}_{\mu}^{i} \mathcal{F}_{\beta \gamma}^{j} \mathcal{F}_{\delta \epsilon}^{k}-\frac{1}{2} V^{-2} \alpha_{i} \alpha^{i} \\
& -\bar{\psi}_{A \alpha} \gamma^{\alpha \beta \gamma} \mathcal{D}_{\beta} \psi_{\gamma}^{A}-\bar{\zeta}_{A} \gamma^{\beta} \mathcal{D}_{\beta} \zeta^{A}-G^{\perp}{ }_{i j} \bar{\lambda}_{A}^{i} \gamma^{\beta} \mathcal{D}_{\beta} \lambda^{A j} \\
& +\frac{i \sqrt{2}}{16}\left(\bar{\psi}_{A \gamma} \gamma^{\alpha \beta \gamma \delta} \psi_{\delta}^{A}+2 \bar{\psi}_{A}^{\alpha} \psi^{A \beta}-G^{\perp}{ }_{i j} \bar{\lambda}_{A}^{i} \gamma^{\alpha \beta} \lambda^{A j}-\bar{\zeta}_{A} \gamma^{\alpha \beta} \zeta^{A}\right) b_{k} \mathcal{F}_{\alpha \beta}^{k} \\
& +\frac{\sqrt{2}}{8} G^{\perp}{ }_{i j} \bar{\lambda}_{A}^{i} \gamma^{\alpha} \gamma^{\beta \gamma} \psi_{\alpha}^{A} \mathcal{F}_{\beta \gamma}^{j}-\frac{i \sqrt{2}}{16} \mathcal{K}^{\perp}{ }_{i j k} \bar{\lambda}_{A}^{i} \gamma^{\alpha \beta} \lambda^{A j} \mathcal{F}_{\alpha \beta}^{k} \\
& -\frac{i}{2} G^{\perp}{ }_{i j} \bar{\lambda}_{A}^{i} \gamma^{\alpha} \gamma^{\beta} \psi_{\alpha}^{A} \partial_{\alpha} b^{j}+\frac{i \sqrt{2}}{2} \bar{\zeta}_{A} \gamma^{\alpha} \gamma^{\beta} \psi_{\alpha}^{B} E^{A}{ }_{B \beta} \\
& +\frac{\sqrt{2}}{4} V^{-1}{ }_{\alpha \tau^{A}}{ }_{B} \bar{\psi}_{A \alpha} \gamma^{\alpha \beta} \psi_{\beta}^{B}+\frac{i \sqrt{2}}{2} V^{-1} \alpha_{i}^{\perp} \tau^{A}{ }_{B} \bar{\lambda}_{A}^{i} \gamma^{\alpha} \psi_{\alpha}^{B}-2 i V^{-1} \alpha \tau^{A}{ }_{B} \bar{\zeta}_{A} \gamma^{\alpha} \psi_{\alpha}^{B} \\
& +\frac{\sqrt{2}}{12} V^{-1}\left(9 \mathcal{K}^{-1} \mathcal{K}^{\perp}{ }_{i j k}+G^{\perp}{ }_{i j} b_{k}\right) \alpha^{k} \tau^{A}{ }_{B} \bar{\lambda}_{A}^{i} \lambda^{B j}+V^{-1} \alpha_{i}^{\perp} \tau^{A}{ }_{B} \bar{\zeta} \lambda^{B i} \\
& +\frac{\sqrt{2}}{4} V^{-1} \alpha \tau^{A}{ }_{B} \bar{\zeta}_{A} \zeta^{B},
\end{aligned}
$$

where $\alpha=\alpha_{i} b^{i}$, and the derivatives are given by Eqs. (35] 38). The supergravity connection $\Omega$ contains fermionic torsion terms which can be determined in the 1.5 order formalism by an independent variation of $\Omega$ in the Lagrangian.

The boundary contributions to the supergravity Lagrangian are

$$
\begin{aligned}
& \mathcal{L}_{0}\left(\partial \mathcal{M}_{1}\right)=K-\frac{\sqrt{2}}{2} V^{-1} \alpha-\frac{1}{4} \tau^{A}{ }_{B} \bar{\psi}_{A \mu} \gamma^{\mu \nu} \bar{\psi}_{\nu}^{B}-\frac{1}{4} \tau^{A}{ }_{B} \bar{\zeta}_{A} \zeta^{B}-\frac{1}{4} \tau^{A}{ }_{B} G_{i j} \bar{\lambda}_{A}^{i} \lambda^{B j}+\mathcal{T} \\
& \mathcal{L}_{0}\left(\partial \mathcal{M}_{2}\right)=K+\frac{\sqrt{2}}{2} V^{-1} \alpha+\frac{1}{4} \tau^{A}{ }_{B} \bar{\psi}_{A \mu} \gamma^{\mu \nu} \bar{\psi}_{\nu}^{B}+\frac{1}{4} \tau^{A}{ }_{B} \bar{\zeta}_{A} \zeta^{B}+\frac{1}{4} \tau^{A}{ }_{B} G_{i j}^{\perp} \bar{\lambda}_{A}^{i} \lambda^{B j}+\mathcal{T}
\end{aligned}
$$

where $\mathcal{T}$ is a torsion term which cancels a total derivative in $R(\Omega)$. The matter Lagrangian on the $E_{6}$ boundary $\partial \mathcal{M}_{1}$ is

$$
\begin{aligned}
\mathcal{L}_{Y M}\left(\partial \mathcal{M}_{1}\right)= & -\frac{1}{4} V F_{\mu \nu}^{I} F^{I \mu \nu}-\frac{1}{2} \bar{\chi}_{A}^{I} \gamma^{\mu} D_{\mu} \chi^{I A} \\
& -2 G_{i j} \mathcal{D}_{\mu} C^{i p} \mathcal{D}^{\mu} \bar{C}^{j}{ }_{p}-\frac{1}{2} G_{i j} \bar{\eta}_{A}^{i p} \gamma^{\mu} \mathcal{D}_{\mu} \eta^{A j}{ }_{p} \\
& -\frac{1}{2} V^{-1} G^{i j} \partial_{C^{i}} W \partial_{\bar{C}^{j}} \bar{W}-2 V^{-1} \bar{C}^{i} \Lambda^{I} C_{i} \bar{C}^{j} \Lambda^{I} C_{j} \\
& -2 V^{-1 / 2} G_{i j}\left(\bar{\chi}_{L}^{I} \eta_{L}{ }^{i}{ }_{q} C^{j p} \Lambda^{I q}{ }_{p}+\bar{\chi}_{R}^{I} \eta_{R}{ }^{i p} \bar{C}^{j}{ }_{q} \Lambda^{I q}{ }_{p}\right) \\
& +\frac{\sqrt{3}}{2} V^{-1 / 2} \mathcal{K}^{-1} \mathcal{K}_{i j k}\left(d_{p q r} C^{k r} \bar{\eta}_{L}^{i p} \eta_{L}{ }^{j q}+d^{p q r} \bar{C}^{k}{ }_{r} \bar{\eta}_{R p}^{i} \eta_{R}{ }^{j}{ }_{q}\right) \\
& -\bar{\psi}_{A \mu} j^{A \mu}-\bar{\zeta}_{A} j^{A}-G_{i j}^{\perp} \bar{\lambda}_{A}^{i} j^{A j}-\Theta^{\mu} \mathcal{D}_{\mu} \sigma .
\end{aligned}
$$

The chiral compoents are $\chi_{L}=\chi^{1}, \chi_{R}=-\chi^{2}, \eta_{L}{ }^{i p}=\eta^{2 i p}$ and $\eta_{R}^{i p}=\eta^{1 i p}$. The bulk fields couple to supercurrents 
and a topological current given by

$$
\begin{aligned}
j^{A \mu}= & \frac{1}{4} V^{1 / 2} \gamma^{\rho \sigma} \gamma^{\mu} F^{I}{ }_{\rho \sigma} \chi^{A I}+V^{-1 / 2} \tau^{A}{ }_{B} \bar{C}^{i} \Lambda^{I} C_{i} \gamma^{\mu} \chi^{B I} \\
& +\frac{\sqrt{3}}{2} V^{-1 / 2} \mathcal{K}^{-1} \mathcal{K}_{i j k} d_{p q r} \gamma^{\mu}\left(C^{i p} C^{j q} P_{L} \eta^{A k r}+\bar{C}^{i p} \bar{C}^{j q} P_{R} \eta^{A k r}\right) \\
& +\gamma^{\beta} \gamma^{\mu}\left(D_{\beta} C^{i p} P_{R} \eta^{A}{ }_{i p}+D_{\beta} \bar{C}^{i p} P_{L} \eta^{A}{ }_{i p}\right), \\
j^{A}= & i \frac{\sqrt{2}}{4} V^{1 / 2} \gamma^{\rho \sigma} F^{I}{ }_{\rho \sigma} \chi^{A I}-i \frac{3 \sqrt{2}}{2} V^{-1 / 2} \tau^{A}{ }_{B} \bar{C}^{i} \Lambda^{I} C_{i} \chi^{B I} \\
& -i 5 \sqrt{6} V^{-1 / 2} \mathcal{K}^{-1} \mathcal{K}_{i j k} d_{p q r}\left(C^{i p} C^{j q} P_{L} \eta^{A k r}+\bar{C}^{i p} \bar{C}^{j q} P_{R} \eta^{A k r}\right), \\
j_{i}^{A}= & -i V^{-1 / 2}\left(6 \mathcal{K}^{-1} \mathcal{K}_{i j k}+4 G_{i j} b_{k}\right) \tau^{A}{ }_{B} \bar{C}^{j} \Lambda^{I} C^{k} \chi^{B I} \\
& +2 i\left(6 \mathcal{K}^{-1} \mathcal{K}_{i j k}+4 G_{i j} b_{k}\right) \gamma^{\alpha}\left(D_{\alpha} C^{j p} P_{R} \eta^{A k}{ }_{p}+D_{\alpha} \bar{C}^{j p} P_{L} \eta^{A k}{ }_{p}\right) \\
\Theta^{\mu}= & \frac{1}{12} \epsilon^{\mu \nu \rho \sigma}\left(\omega_{\nu \rho \sigma}^{Y}+\frac{1}{4} V^{-1} \bar{\chi}_{A}^{I} \gamma_{\nu \rho \sigma} \chi^{A I}\right. \\
& \left.+\frac{1}{4} V^{-1} G_{i j}\left(\bar{\eta}_{R p}^{i} \gamma_{\mu \nu \rho} \eta_{L}^{j p}+\bar{\eta}_{L}^{i p} \gamma_{\mu \nu \rho} \eta_{R}^{j}\right)\right) .
\end{aligned}
$$

On the $E_{8}$ boundary,

$$
\mathcal{L}_{Y M}\left(\partial \mathcal{M}_{2}\right)=-\frac{1}{4} V F_{\mu \nu}^{I} F^{I \mu \nu}-\frac{1}{2} \bar{\chi}_{A}^{I} \gamma^{\mu} D_{\mu} \chi^{I A}-\bar{\psi}_{A \mu} j^{A \mu}-\bar{\zeta}_{A} j^{A}+\Theta^{\mu} \mathcal{D}_{\mu} \sigma .
$$

The currents are the same as above, with the matter fields $C^{i p}$ and $\eta^{A i p}$ set to zero.

\section{Appendix D: Supersymmetry transformations}

We start from the 11-dimensional supersymmetry transformations from Green et al. [31]. We shall denote the supersymmetry parameter in five dimensions by $s^{A}$. Products of three or more fermion fields have been dropped. After reduction, the supersymmetry transformations of the bulk supergravity fields become

$$
\begin{aligned}
\delta e^{\hat{\alpha}}{ }_{\alpha} & =\frac{1}{2} \bar{s}_{A} \gamma^{\hat{\alpha}} \psi_{\alpha}^{A}, \\
\delta \psi_{\alpha}^{A} & =\mathcal{D}_{\alpha} s^{A}+i \frac{\sqrt{2}}{6}\left(\gamma_{\alpha}{ }^{\beta \gamma}-4 \delta_{\alpha}{ }^{\beta \gamma}\right) b_{i} \mathcal{F}_{\beta \gamma}^{i} s^{A}+\frac{1}{6} V^{-1} \alpha \tau^{A}{ }_{B} \gamma_{\alpha} \eta^{B}, \\
\delta \mathcal{A}_{\alpha} & =\frac{i \sqrt{6}}{4} \bar{s}_{A} \psi_{\alpha}^{A} .
\end{aligned}
$$

The derivative $\mathcal{D}_{\alpha}$ is the same as the derivative which acts on the gravitino in Eq. (36). For the hypermultiplet,

$$
\begin{aligned}
\delta V & =-i \frac{\sqrt{2}}{2} V \bar{s}_{A} \zeta^{A}, \\
\delta \sigma & =-\frac{\sqrt{2}}{2}\left(V \tau^{A}{ }_{B} \bar{s}_{A} \zeta^{B}-V^{1 / 2} \bar{\xi}_{2} \zeta^{1}-V^{1 / 2} \xi \bar{s}_{1} \zeta^{2}\right), \\
\delta \xi & =-i \frac{\sqrt{2}}{2} V^{1 / 2} \bar{s}_{2} \zeta^{1} \\
\delta \zeta & =-i \frac{\sqrt{2}}{4} E^{A}{ }_{B \alpha} \gamma^{\alpha} s^{B}
\end{aligned}
$$

and for the Abelian multiplets,

$$
\begin{aligned}
\delta \mathcal{A}^{\perp i} & =0 \\
\delta b^{i} & =\frac{i}{2} \bar{s}_{A} \lambda^{A i \perp} \\
\delta \lambda^{\perp i} & =\frac{i}{2} \partial_{\alpha} b^{i} \gamma^{\alpha} s^{A}+\frac{\sqrt{2}}{2} \gamma^{\alpha \beta} \mathcal{F}_{\alpha \beta}^{\perp i} s^{A}-G^{\perp i j} \mathcal{P}_{j}{ }^{A}{ }_{B} s^{B} .
\end{aligned}
$$


On the boundaries, the supersymmetry parameter is a Majorana spinor with chiral components $s_{L}=s^{1}$ and $s_{R}=-s^{2}$. The supersymmetry transformations of the fields on the $E_{6}$ boundary become

$$
\begin{aligned}
\delta A_{\mu}^{I} & =\frac{1}{2} V^{-1 / 2} \bar{s}_{A} \gamma_{\mu} \chi^{A I}, \\
\delta \chi^{A I} & =-\frac{1}{4} V^{1 / 2} F_{\mu \nu}^{I} \gamma^{\mu \nu} s^{A}+i V^{-1 / 2} G_{i j} \bar{C}^{i} \Lambda^{I} C^{j} \tau^{A}{ }_{B} s^{B}, \\
\delta C^{i p} & =\frac{1}{2} \bar{s}_{L} \eta_{L}{ }^{i p}-\frac{1}{4} i \Gamma^{i}{ }_{j k} C^{j p} \bar{s}_{A} \lambda^{A k \perp}, \\
\delta \eta_{L}{ }^{i p} & =-\mathcal{D}_{\mu} C^{i p} \gamma^{\mu} s_{R}-\sqrt{3} \mathcal{K}^{-1} \mathcal{K}^{i}{ }_{j k} d^{p q r} \bar{C}^{j}{ }_{q} \bar{C}^{k}{ }_{r} s_{L}
\end{aligned}
$$

where $\mathcal{D}_{\mu} C^{i p}$ is defined in Eq. (51). These reduce to the standard supersymmetry transformations of the Yang-Mills and chiral multiplets when $V=1$.

[1] P. Horava and E. Witten, Nucl. Phys. B460, 506 (1996), hep-th/9510209.

[2] P. Horava and E. Witten, Nucl. Phys. B475, 94 (1996), hep-th/9603142.

[3] E. Witten, Nucl. Phys. B471, 135 (1996), hep-th/9602070.

[4] T. Banks and M. Dine, Nucl. Phys. B479, 173 (1996), hep-th/9605136.

[5] R. Donagi, Y.-H. He, B. A. Ovrut, and R. Reinbacher, JHEP 12, 054 (2004), hep-th/0405014.

[6] R. Donagi, Y.-H. He, B. A. Ovrut, and R. Reinbacher, Phys. Lett. B598, 279 (2004), hep-th/0403291.

[7] L. B. Anderson, J. Gray, D. Grayson, Y.-H. He, and A. Lukas, Commun. Math. Phys. 297, 95 (2010), 0904.2186.

[8] L. B. Anderson, J. Gray, Y.-H. He, and A. Lukas, JHEP 02, 054 (2010), 0911.1569.

[9] N. Ahmed and I. G. Moss, Nucl. Phys. B833, 133 (2010), 0907.1602.

[10] G. Curio, A. Krause, and D. Lust, Fortsch. Phys. 54, 225 (2006), hep-th/0502168.

[11] V. Braun and B. A. Ovrut, JHEP 07, 035 (2006), hep-th/0603088.

[12] L. B. Anderson, J. Gray, A. Lukas, and B. Ovrut, Phys. Rev. D83, 106011 (2011), 1102.0011.

[13] A. Lukas, B. A. Ovrut, K. S. Stelle, and D. Waldram, Nucl. Phys. B552, 246 (1999), hep-th/9806051.

[14] A. Lukas, B. A. Ovrut, K. S. Stelle, and D. Waldram, Phys. Rev. D59, 086001 (1999), hep-th/9803235.

[15] I. G. Moss, Phys. Lett. B577, 71 (2003), hep-th/0308159.

[16] I. G. Moss, Nucl. Phys. B729, 179 (2005), hep-th/0403106.

[17] I. G. Moss, Phys. Lett. B637, 93 (2006), hep-th/0508227.

[18] P. Horava, Phys. Rev. D54, 7561 (1996), hep-th/9608019.

[19] N. Ahmed and I. G. Moss, JHEP 12, 108 (2008), 0809.2244.

[20] D. V. Belyaev and P. van Nieuwenhuizen, JHEP 02, 047 (2008), 0711.2272.

[21] D. V. Belyaev, JHEP 01, 047 (2006), hep-th/0509172.

[22] T. G. Pugh, E. Sezgin, and K. S. Stelle, JHEP 02, 115 (2011), 1008.0726.

[23] M. Fabinger and P. Horava (2000), hep-th/0002073.

[24] S. Weinberg, The quantum theory of fields. Vol. 3: Supersymmetry (Cambridge, UK: Univ. Pr., 2002).

[25] H. C. Luckock and I. G. Moss, Class Quantum grav 6, 1993 (1989).

[26] I. G. Moss, JHEP 11, 067 (2008), 0810.1662.

[27] I. G. Moss and P. J. Silva, Phys. Rev. D55, 1072 (1997), gr-qc/9610023.

[28] I. G. Moss and S. J. Poletti, Phys. Lett. B333, 326 (1994), gr-qc/9405044.

[29] I. G. Moss and J. P. Norman, JHEP 09, 020 (2004), hep-th/0401181.

[30] P. Candelas and X. de la Ossa, Nucl. Phys. B355, 455 (1991).

[31] M. B. Green, J. H. Schwarz, and E. Witten, Superstring theory. Vol. 2: Loop amplitudes, anomalies and phenomenology (Cambridge, UK: Univ. Pr., 1987), ( Cambridge Monographs On Mathematical Physics). 